\title{
Dacron $^{\circledR}$ vs. PTFE as bypass materials in peripheral vascular surgery - systematic review and meta-analysis Stephanie Roll*1, Jacqueline Müller-Nordhorn¹, Thomas Keil1, Hans Scholz², Daniela Eidt ${ }^{3}$, Wolfgang Greiner ${ }^{4}$ and Stefan N Willich ${ }^{1}$
}

\begin{abstract}
Address: ${ }^{1}$ Institute for Social Medicine, Epidemiology and Health Economics, Charité University Medical Center, Berlin, Germany, ${ }^{2}$ Department of Vascular Surgery, Queen Elisabeth Hospital, Berlin, Germany, ${ }^{3}$ Centre for Health Economics and Health System Research, University of Hanover, Germany and ${ }^{4}$ Department of Health Economics and Health Management, University of Bielefeld, Germany

Email: Stephanie Roll* - stephanie.roll@charite.de; Jacqueline Müller-Nordhorn - jacqueline.mueller-nordhorn@charite.de; Thomas Keil - thomas.keil@charite.de; Hans Scholz - h.scholz@keh-berlin.de; Daniela Eidt - de@insurance.ivbl.uni-hannover.de; Wolfgang Greiner - wolfgang.greiner@uni-bielefeld.de; Stefan N Willich - stefan.willich@charite.de

* Corresponding author
\end{abstract}

Published: 19 December 2008

BMC Surgery 2008, 8:22 doi:10.1/86/147|-2482-8-22

This article is available from: http://www.biomedcentral.com/147I-2482/8/22

(C) 2008 Roll et al; licensee BioMed Central Ltd.

This is an Open Access article distributed under the terms of the Creative Commons Attribution License (http://creativecommons.org/licenses/by/2.0), which permits unrestricted use, distribution, and reproduction in any medium, provided the original work is properly cited.
Received: 26 August 2008

Accepted: 19 December 2008

\begin{abstract}
Background: In peripheral vascular bypass surgery different synthetic materials are available for bypass grafting. It is unclear which of the two commonly used materials, polytetrafluoroethylene (PTFE) or polyester (Dacron ${ }^{\circledR}$ ) grafts, is to be preferred. Thus, the aim of this meta-analysis and systematic review was to compare the effectiveness of these two prosthetic bypass materials (Dacron ${ }^{\circledR}$ and PTFE).
\end{abstract}

Methods: We performed a systematic literature search in MEDLINE, Cochrane-Library CENTRAL, EMBASE and other databases for relevant publications in English and German published between 1999 and 2008. Only randomized controlled trials were considered for inclusion. We assessed the methodological quality by means of standardized checklists. Primary patency was used as the main endpoint. Random-effect meta-analysis as well as pooling data in life table format was performed to combine study results.

Results: Nine randomized controlled trials (RCT) were included. Two trials showed statistically significant differences in primary patency, one favouring Dacron ${ }^{\circledR}$ and one favouring PTFE grafts, while 7 trials did not show statistically significant differences between the two materials. Metaanalysis on the comparison of PTFE vs. Dacron ${ }^{\circledR}$ grafts yielded no differences with regard to primary patency rates (hazard ratio 1.04 (95\% confidence interval $[0.85 ; 1.28])$, no significant heterogeneity $\left.\left(p=0.32,1^{2}=14 \%\right)\right)$. Similarly, there were no significant differences with regard to secondary patency rates.

Conclusion: Systematic evaluation and meta-analysis of randomized controlled trials comparing Dacron ${ }^{\circledR}$ and PTFE as bypass materials for peripheral vascular surgery showed no evidence of an advantage of one synthetic material over the other. 


\section{Background}

The prevalence of symptomatic peripheral arterial disease in the adult population ranges between $0.6 \%$ and $9.2 \%$ and increases with age $[1,2]$. Patients with peripheral arterial disease have an increased risk of cardiovascular morbidity and mortality, showing a similar risk factor profile to patients with other atherosclerotic diseases. In the nonpharmacological treatment of symptomatic peripheral arterial disease, peripheral vascular surgical interventions such as bypass grafting and endarterectomy play an important role [3]. The long-term aim of surgical interventions is to prevent amputation of the limb and to reduce its resulting disability. According to current guidelines, surgical interventions are indicated for individuals with symptomatic disease (claudication), significant functional disability, resistance to exercise or pharmacotherapy, and a reasonable likelihood of symptomatic improvement [4]. Whereas endarterectomy is an option in strictly localised disease, bypass grafting is generally used to circumvent severely stenosed sections of the peripheral arteries.

Different materials can be used for bypass grafting including autologous and homologous grafts from the saphenous vein or the human umbilical vein as well as prosthetic graft materials such as polytetrafluoroethylene (PTFE) or polyester $\left(\right.$ Dacron $^{\circledR}$ ) grafts. Most studies so far have shown that autologous vein is superior to prosthetic graft materials in bypass surgery [5-7]. A recent review comparing venous and PTFE bypass procedures reported 5-year primary patency rates of $74 \%$ and $39 \%$, respectively [8]. However, almost a third of patients eligible for peripheral bypass procedures do not have suitable veins, making the use of prosthetic materials necessary [9]. Also, due to the high prevalence of cardiovascular co-morbidity, it may be required to keep suitable autologous veins for potential future use in coronary artery bypass grafting. The objective of our systematic review was, therefore, to identify available evidence and compare the effectiveness of the prosthetic bypass materials Dacron ${ }^{\circledast}$ and PTFE in peripheral vascular bypass surgery and to perform meta-analyses, if possible.

\section{Methods}

\section{Literature search}

A trained librarian performed a comprehensive systematic literature search for relevant publications using the following databases: AMED, BIOSIS Previews, CAB Abstracts, CATFILEplus (CATLINE), Cochrane Library CDSR, Cochrane Library - CENTRAL, Elsevier BIOBASE, EMBASE, EMBASE Alert, ETHMED, GeroLit, GLOBAL Health, HECLINET, IPA, MEDLINE Alert, MEDLINE, NHS-CRD-DARE, NHS-CRD-HTA (INAHTA), NHS-EED, SciSearch, and SOMED. The search terms included "bypass", "revascularization", "artery reconstruction", "graft", "prosthesis", and "material". The search was performed in February 2005 with an update search performed in MEDLINE and CENTRAL (Cochrane Central Register of Controlled Trials) for publications until August 2008. The systematic database search was supplemented by manual search of reference lists of included articles. The inclusion criteria of studies were: (i) randomized controlled trial (RCT) as study design; (ii) comparison of polytetrafluoroethylene (PTFE) or polyester $\left(\right.$ Dacron $^{\circledast}$ ) grafts for peripheral vascular bypass surgery; (iii) publication in English or German; and (iv) publication from 1999 to date. We focused on publications of English language to cover the most important and qualitatively high trials therewith (we additionally searched for articles in German for a potential adaption to the situation in Germany). We included studies published in and after 1999 as the purpose of this review was to provide an overview focusing on the present evidence from more recent trials.

Excluded were studies due to the following criteria: (i) case series; (ii) retrospective studies; (iii) studies comparing venous vs. prosthetic graft materials.

\section{Methodological assessment and endpoints}

The methodological quality of relevant publications was assessed using standardized checklists developed by the German Scientific Working Group "Technology Assessment for Health Care". [10] evaluating the selection process of patients, randomization procedure, assessment of outcomes, drop-out rates, and adequate statistical methods. The primary outcome for this review was primary patency, as defined by the authors. According to Rutherford et al. primary patency should be assessed by objective methods such as vascular imaging techniques, palpable pulse, biphasic or triphasic Doppler, segmental limb pressure index, etc. [11]. We here present primary patency rates as defined and reported in each trial. Secondary outcomes were secondary patency, graft infection rates, limb salvage or amputation rates, and perioperative ( $<30$ days) mortality as presented in each study.

\section{Statistical analysis}

To perform a meta-analysis, study data from accumulated life tables was extracted from each study where available. If failure data was given for one or three months intervals it was combined to yield 6-months interval data for all studies. Time of event (graft failure) or censoring (withdrawals) was thus assigned to the end of each 6-months interval. A Cox proportional hazard model was used to calculate hazard ratios with their 95\% confidence intervals (CI) and standard errors (SE) for the independent variable "material". This was done for each study separately. In a random-effect meta-analysis these hazard ratios were then combined with weights according to their standard 
error (inverse variance method), yielding an overall hazard ratio (with 95\% CI). Heterogeneity was tested using the chi-squared Q-statistic and inconsistency was quantified by $\mathrm{I}^{2}$. Life table data was also pooled to provide Kaplan-Meier graphs on graft failure events. All analyses were performed with SAS V9.1.3 (SAS Institute Inc., Cary, NC, USA) and Review Manager Version 5.0 (Copenhagen: The Nordic Cochrane Centre, The Cochrane Collaboration, 2008).

\section{Results}

We identified a total of 4421 publications in the search process. Excluding duplicates and non-relevant publications based on their title and/or abstract resulted in 419 publications for further quality assessment (Fig. 1). Of these, 9 publications were found to be relevant to the research question and fulfilled all inclusion criteria [12-20].

All 9 RCT included in our analysis used primary patency as their main outcome measure. All studies provided a definition for the term patency in their methods section, with primary patency usually meaning unassisted/uninterrupted patency with no follow-up procedures on the bypass assessed by objective methods; one study, however, defined primary patency as assisted primary patency [12]. Seven studies also reported results on secondary patency rates. Five studies present limb salvage or amputation rates, 4 perioperative mortality ( $<30$ days), and 6 graft infection as further outcomes. Most of these endpoints were not defined in detail.

Table 1 gives an overview of study characteristics and main results of all RCT included in our analysis.

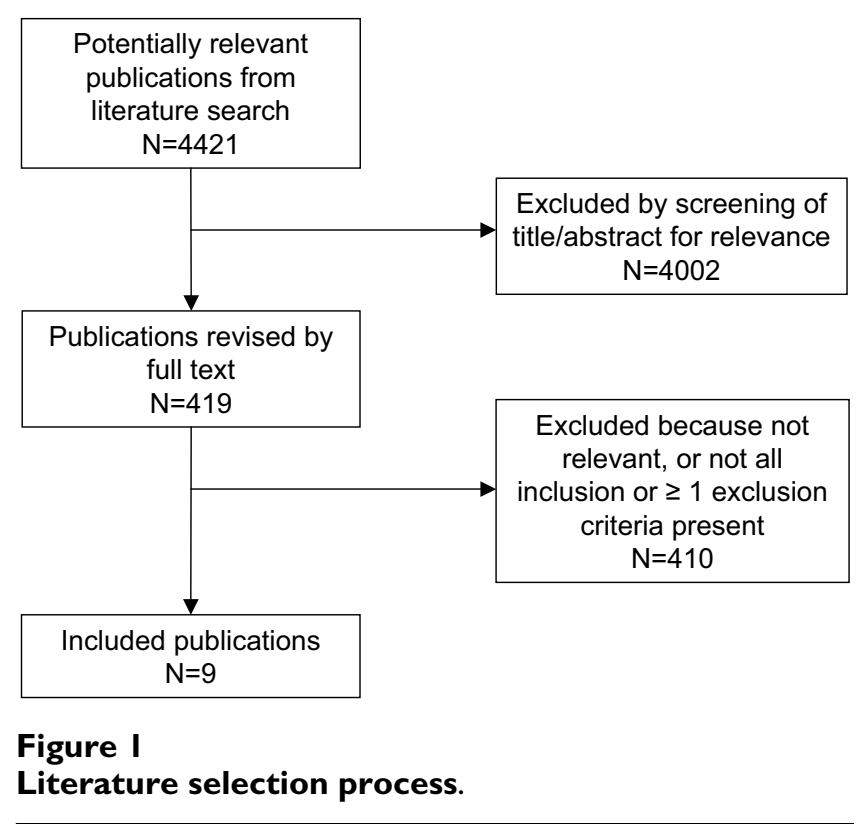

Of the 9 included studies, 7 studies showed no significant differences between Dacron ${ }^{\circledast}$ and PTFE regarding primary patency, 1 study showed significantly higher patency rates for Dacron ${ }^{\circledast}$ after 2 years [20], and 1 study showed significantly higher patency rates for PTFE after 2 years [17].

The 7 RCT reporting results on secondary patency yielded results similar to primary patency rates, e.g. no significant differences between the prosthetic materials were found in 6 studies, and 1 study showed significantly higher secondary patency after 2 years with Dacron ${ }^{\circledR}$ grafts. Of the studies reporting results regarding limb salvage or amputation rates, 4 showed no significant difference, whereas 1 showed a significantly better rate for Dacron ${ }^{\circledast}$ grafts. None of the studies found significant differences in terms of graft infection or perioperative mortality (<30 days).

\section{Meta-analysis}

Meta-analysis was performed for the comparison of Dacron $^{\circledR}$ vs. PTFE on primary patency for the 5 studies where adequate data was available $[12-14,17,18]$. Two studies provided also data on secondary patency $[13,17]$. The combined hazard ratios for PTFE vs. Dacron ${ }^{\circledR}$ were $1.04(95 \%$ CI $[0.85 ; 1.28])$ for primary patency (Fig. 2) and 1.02 (95\% CI $[0.65 ; 1.62])$ for secondary patency (Fig. $3)$. There was no significant heterogeneity between the studies $(\mathrm{p}=0.32$ and $\mathrm{p}=0.24)$. The Kaplan-Meier curves of the pooled data reflect the similar efficacy of the materials regarding both primary and secondary patency (Fig. 4 and Fig. 5).

\section{Discussion}

The present meta-analysis indicated that there are no major differences in primary and secondary patency rates between the two prosthetic graft materials Dacron ${ }^{\circledast}$ and PTFE. Of the 9 included studies, one study showed statistically significant higher patency rates for Dacron ${ }^{\circledR}$ after 2 years [20], 1 study showed statistically significant higher patency rates for PTFE after 2 years [17], while 7 studies showed no statistically significant differences between the two materials regarding primary patency $[12-16,18,19]$.

Our study complements a systematic Cochrane review on femoro-popliteal bypass surgery by Mamode and Scott comparing saphenous vein graft with PTFE or Dacron ${ }^{\circledast}$, human umbilical vein with PTFE, and PTFE vs. Dacron ${ }^{\circledR}$ [21]. However, only one study comparing PTFE with Dacron ${ }^{\circledast}$ in above-knee popliteal grafting was identified [22]. This study by Abbott et al. was published before the search period of our review. It did not show a significant difference regarding primary or secondary patency rates at the 3-year follow-up.

One of the studies in the present review compared gelatine-coated Dacron ${ }^{\circledast}$ vs. collagen-coated Dacron ${ }^{\circledast}$ showing 
Table I: Overview of included studies of Dacron $^{\circledR}$ vs. PTFE as bypass materials in peripheral vascular surgery

\begin{tabular}{|c|c|c|c|c|c|c|c|c|c|c|c|}
\hline \multirow[t]{2}{*}{ Author } & \multirow[t]{2}{*}{ Year } & \multirow[t]{2}{*}{ Indication } & \multirow{2}{*}{$\begin{array}{l}\text { Site of } \\
\text { bypass }\end{array}$} & \multirow{2}{*}{$\begin{array}{l}\text { Intervent } \\
\text { ion }\end{array}$} & \multirow{2}{*}{$\begin{array}{l}\text { Addition } \\
\text { al } \\
\text { intervent } \\
\text { ion }\end{array}$} & \multirow{2}{*}{$\begin{array}{c}\text { Follow- } \\
\text { up } \\
\text { (years) }\end{array}$} & \multicolumn{2}{|c|}{$\mathbf{N}^{*}$} & \multirow{2}{*}{$\begin{array}{l}\text { Primary } \\
\text { patency } \\
(\mathrm{N}= \\
\text { number } \\
\text { of patients } \\
\text { or grafts } \\
\text { under } \\
\text { risk) }\end{array}$} & \multirow{2}{*}{$\begin{array}{l}\text { Superior } \\
\text { primary } \\
\text { patency }\end{array}$} & \multirow[t]{2}{*}{ Funding } \\
\hline & & & & & & & Baseline & $\begin{array}{l}\text { End of } \\
\text { study }\end{array}$ & & & \\
\hline $\begin{array}{l}\text { Johnson } \\
\text { and Lee } \\
{[12]}\end{array}$ & 1999 & $\begin{array}{l}\text { disabling } \\
\text { claudication, } \\
\text { rest pain or } \\
\text { tissue } \\
\text { necrosis }\end{array}$ & $\begin{array}{l}\text { femorof } \\
\text { emoral, } \\
\text { axillofe } \\
\text { moral, } \\
\text { axillobife } \\
\text { moral }\end{array}$ & $\begin{array}{l}\text { Dacron }^{\circledR} \\
\text { vs. PTFE }\end{array}$ & $\begin{array}{l}\text { Aspirin } \\
650 \mathrm{mg} / \mathrm{d}\end{array}$ & 5 & 419 & 47 & $\begin{array}{l}\text { At I, 3, } 5 \\
\text { years: } \\
\text { - Dacron }{ }^{\circledR}: \\
79 \%, 63 \%, \\
50 \%(\mathrm{~N}= \\
\text { I25, 73, 32) } \\
\text { - PTFE: } \\
77 \%, 62 \%, \\
47 \%(\mathrm{~N}= \\
103,53,15)\end{array}$ & $\begin{array}{l}\text { n.s. } \\
\text { (p-value } \\
\text { not } \\
\text { reported) }\end{array}$ & n.r. \\
\hline $\begin{array}{l}\text { Robinso } \\
\text { n et al. } \\
{[13]}\end{array}$ & 1999 & $\begin{array}{l}\text { disabling } \\
\text { claudication, } \\
\text { rest pain or } \\
\text { tissue } \\
\text { necrosis }\end{array}$ & $\begin{array}{l}\text { femorop } \\
\text { opliteal } \\
\text { (above- } \\
\text { knee } \\
\text { and } \\
\text { below- } \\
\text { knee) }\end{array}$ & $\begin{array}{l}\text { gelatine- } \\
\text { sealed } \\
\text { Dacron }^{\circledR} \\
\text { vs. PTFE }\end{array}$ & $\begin{array}{l}\text { Cephalothi } \\
\text { n, Heparin, } \\
\text { Aspirin }\end{array}$ & 3 & 108 & 19 & $\begin{array}{l}\text { At I, } 2,3 \\
\text { years: } \\
\text { - Dacron }{ }^{\circledR}: \\
70 \%, 56 \% \text {, } \\
47 \% \\
(\mathrm{~N}=27,18 \text {, } \\
9) \\
\text { - PTFE: } \\
72 \%, 52 \%, \\
52 \% \\
(\mathrm{~N}=33,16, \\
10)\end{array}$ & $\begin{array}{l}\text { n.s. } \\
(p=0.87)\end{array}$ & n.r. \\
\hline $\begin{array}{l}\text { Green et } \\
\text { al. [14] }\end{array}$ & 2000 & $\begin{array}{l}\text { superficial } \\
\text { femoral } \\
\text { artery } \\
\text { occlusion }\end{array}$ & $\begin{array}{l}\text { femorop } \\
\text { opliteal } \\
\text { (above- } \\
\text { knee) }\end{array}$ & $\begin{array}{l}\text { collagen- } \\
\text { impregnat } \\
\text { ed } \\
\text { Dacron }{ }^{\circledR} \\
\text { vs. ePTFE }\end{array}$ & n.r. & 5 & 240 & 10 & $\begin{array}{l}\text { At I, 3, } 5 \\
\text { years:: } \\
\text { - Dacron }{ }^{\circledR}: \\
78 \%, 65 \% \text {, } \\
45 \% \\
(\mathrm{~N}=65,25 \text {, } \\
5) \\
\text { - PTFE: } \\
80 \%, 63 \% \text {, } \\
43 \% \\
(\mathrm{~N}=66,21 \text {, } \\
5)\end{array}$ & $\begin{array}{l}\text { n.s. } \\
\text { (p-value } \\
\text { not } \\
\text { reported) }\end{array}$ & $\begin{array}{l}\text { manufact } \\
\text { urer of } \\
\text { Dacron }^{\circledR}\end{array}$ \\
\hline $\begin{array}{l}\text { Post et } \\
\text { al. [15] }\end{array}$ & 2001 & $\begin{array}{l}\text { indication } \\
\text { for artificial } \\
\text { graft of at } \\
\text { least } 20 \mathrm{~cm} \\
\text { length }\end{array}$ & $\begin{array}{l}\text { femorop } \\
\text { opliteal } \\
\text { (above- } \\
\text { knee } \\
\text { and } \\
\text { below- } \\
\text { knee) }\end{array}$ & $\begin{array}{l}\text { unsealed } \\
\text { Dacron }{ }^{\circledR} \\
\text { vs. PTFE }\end{array}$ & $\begin{array}{l}\text { Anti- } \\
\text { platelet } \\
\text { drugs, } \\
\text { Heparin or } \\
\text { Coumadin }\end{array}$ & 3 & 194 & 50 & $\begin{array}{l}\text { At } 3 \text { years } \\
\text { ( } \mathrm{N}=\text { grafts } \\
\text { under risk): } \\
\text { - Dacron }{ }^{\circledR} \text { : } \\
64 \%(95 \%- \\
\mathrm{Cl} \\
{[55 \% ; 74 \%] \text {, }} \\
\mathrm{N}=28) \\
\text { - PTFE: } 61 \% \\
(95 \%-\mathrm{Cl} \\
{[49 \% ; 72 \%] \text {, }} \\
\mathrm{N}=22)\end{array}$ & $\begin{array}{l}\text { n.s. } \\
(p=0.89)\end{array}$ & $\begin{array}{l}\text { manufact } \\
\text { urer of } \\
\text { Dacron } \\
\text { and PTFE }\end{array}$ \\
\hline
\end{tabular}


Table I: Overview of included studies of Dacron ${ }^{\circledR}$ vs. PTFE as bypass materials in peripheral vascular surgery (Continued)

\begin{tabular}{|c|c|c|c|c|c|c|c|c|c|c|c|}
\hline $\begin{array}{l}\text { Prager } \\
\text { et al. } \\
{[16]}\end{array}$ & 2003 & $\begin{array}{l}\text { aortoiliac } \\
\text { occlusive } \\
\text { disease }\end{array}$ & $\begin{array}{l}\text { aortoilia } \\
\text { c }\end{array}$ & $\begin{array}{l}\text { gelatine- } \\
\text { coated } \\
\text { Dacron }{ }^{\circledR} \\
\text { vs. } \\
\text { collagen- } \\
\text { coated } \\
\text { Dacron } \\
\text { vs. PTFE }\end{array}$ & $\begin{array}{l}\text { Antibiotics } \\
\text {, Heparin } \\
70 \mathrm{IU} / \mathrm{kg}, \\
\text { Fraxiparine } \\
100 \mathrm{mg} / \mathrm{kg} / \\
\text { d (bid for } \\
\text { patients } \\
\text { with } \\
\text { anastomos } \\
\text { es) }\end{array}$ & 8 & 149 & 35 & $\begin{array}{l}\text { At } 5,8 \\
\text { years: } \\
\text { - C- } \\
\text { Dacron }{ }^{\circledR}: \\
89 \%, 78 \% \\
(\mathrm{~N}=24, \mathrm{I} \text { I }) \\
\text { - G- } \\
\text { Dacron } \\
92 \%, 77 \% \\
(\mathrm{~N}=26, \mathrm{I} \text { I) } \\
\text { - PTFE: } \\
88 \%, 79 \% \\
(\mathrm{~N}=29, \mathrm{I})\end{array}$ & $\begin{array}{l}\text { n.s. } \\
(p>0.8)\end{array}$ & n.r. \\
\hline $\begin{array}{l}\text { Robinso } \\
\mathrm{n} \text { and } \\
\text { Fletcher } \\
{[17]}\end{array}$ & 2003 & $\begin{array}{l}\text { disabling } \\
\text { claudication, } \\
\text { rest pain or } \\
\text { tissue loss }\end{array}$ & $\begin{array}{l}\text { femorop } \\
\text { opliteal } \\
\text { (above- } \\
\text { knee } \\
\text { and } \\
\text { below- } \\
\text { knee) }\end{array}$ & $\begin{array}{l}\text { fluoropoly } \\
\text { mer- } \\
\text { coated } \\
\text { Dacron } \\
\text { vs. PTFE }\end{array}$ & $\begin{array}{l}\text { Cephalothi } \\
\text { n, Heparin, } \\
\text { Aspirin }\end{array}$ & 2 & 129 & 21 & $\begin{array}{l}\text { At 6, } 12,24 \\
\text { month: } \\
\text { - Dacron }{ }^{\circledR}: \\
50 \%, 36 \%, \\
36 \% \\
(\mathrm{~N}=27,17 \text {, } \\
9) \\
-\mathrm{PTFE}: \\
71 \%, 56 \%, \\
47 \% \\
(\mathrm{~N}=43,28, \\
\mathrm{I} 2)\end{array}$ & $\begin{array}{l}\text { PTFE } \\
(p= \\
0.002)\end{array}$ & $\begin{array}{l}\text { manufact } \\
\text { urer of } \\
\text { Dacron }^{\circledR} \\
\text { and PTFE }\end{array}$ \\
\hline $\begin{array}{l}\text { Devine } \\
\text { and } \\
\text { McCollu } \\
\text { m [18] }\end{array}$ & 2004 & $\begin{array}{l}\text { occlusive } \\
\text { arterial } \\
\text { disease } \\
\text { (superficial } \\
\text { femoral or } \\
\text { popliteal } \\
\text { artery) }\end{array}$ & $\begin{array}{l}\text { femorop } \\
\text { opliteal } \\
\text { (above- } \\
\text { knee } \\
\text { and } \\
\text { below- } \\
\text { knee) }\end{array}$ & $\begin{array}{l}\text { collagen- } \\
\text { coated, } \\
\text { heparin- } \\
\text { bonded } \\
\text { Dacron }{ }^{\circledR} \\
\text { vs. PTFE }\end{array}$ & $\begin{array}{l}\text { Aspirin } \\
300 \mathrm{mg} / \mathrm{d}\end{array}$ & 5 & 209 & 45 & $\begin{array}{l}\text { At I, 3, } 5 \\
\text { years: } \\
\text { - Dacron }{ }^{\circledR} \text { : } \\
71 \%, 54 \%, \\
46 \% \\
(\mathrm{~N}=70,45, \\
20) \\
- \text { PTFE: } \\
62 \%, 44 \%, \\
35 \% \\
(\mathrm{~N}=62,42, \\
25)\end{array}$ & $\begin{array}{l}\text { n.s. } \\
(p=0.05)\end{array}$ & n.r. \\
\hline $\begin{array}{l}\text { Eiberg et } \\
\text { al. [19] }\end{array}$ & 2006 & $\begin{array}{l}\text { uni-ilia } \\
\text { occlusive } \\
\text { disease }\end{array}$ & $\begin{array}{l}\text { femorof } \\
\text { emoral }\end{array}$ & $\begin{array}{l}\text { fluoropassi } \\
\text { vated, } \\
\text { gelatine- } \\
\text { sealed } \\
\text { Dacron }^{\circledR} \\
\text { vs. ePTFE }\end{array}$ & n.r. & 2 & 198 & 136 & $\begin{array}{l}\text { At I, } 2 \\
\text { years: } \\
\text { - PTFE: } \\
94 \%, 93 \% \\
(\mathrm{~N}=74,63) \\
\text { - Dacron }{ }^{\circledR}: \\
92 \%, 87 \% \\
(\mathrm{~N}=87,73)\end{array}$ & $\begin{array}{l}\text { n.s. } \\
(p= \\
0.350)\end{array}$ & $\begin{array}{l}\text { manufact } \\
\text { urer of } \\
\text { Dacron }^{\circledR} \\
\text { and PTFE }\end{array}$ \\
\hline $\begin{array}{l}\text { Jensen et } \\
\text { al. [20] }\end{array}$ & 2007 & $\begin{array}{l}\text { chronic } \\
\text { lower limb } \\
\text { ischaemia }\end{array}$ & $\begin{array}{l}\text { femorop } \\
\text { opliteal } \\
\text { (above- } \\
\text { knee) }\end{array}$ & $\begin{array}{l}\text { gelatine- } \\
\text { coated } \\
\text { Dacron } \\
\text { vs. PTFE }\end{array}$ & n.r. & 2 & 413 & 150 & $\begin{array}{l}\text { At } 2 \text { years: } \\
\text { - Dacron }{ }^{\circledR}: \\
70 \% \\
(N=78) \\
\text { - PTFE: } 57 \% \\
(N=72)\end{array}$ & $\begin{array}{l}\text { Dacron }^{\circledR} \\
(p= \\
0.002)\end{array}$ & $\begin{array}{l}\text { manufact } \\
\text { urer of } \\
\text { Dacron }^{\circledR}\end{array}$ \\
\hline
\end{tabular}

PTFE: Polytetrafluoroethylene. ePTFE: expanded Polytetrafluoroethylene. IU: international units. d: day. bid: bis in die. n.s.: not significant. n.r.: not reported. Cl: confidence interval.

* Number of patients or grafts under risk

no differences between the study groups [16]. In another study heparin-bonded Dacron ${ }^{\circledast}$ instead of bare Dacron ${ }^{\circledast}$ graft material was used. The issue of different coatings might in itself affect patency outcomes of Dacron ${ }^{\circledR}$ graft.
However this question cannot adequately be assessed at the moment due to the lack of data.

Only 5 studies presented data in adequate life table format for performing a meta-analysis. Results of the meta- 


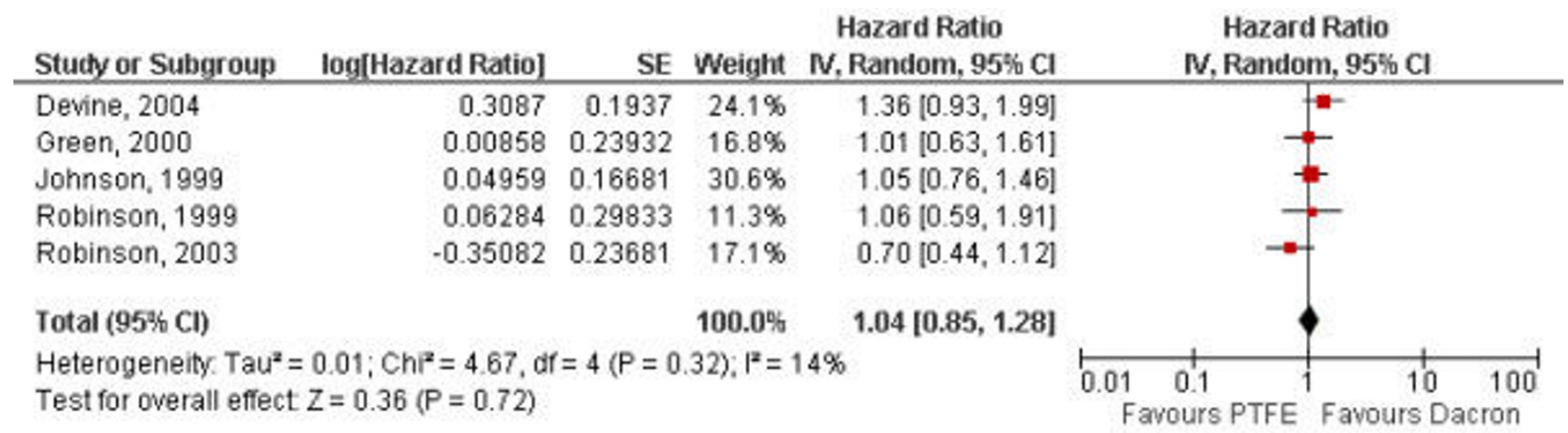

Figure 2

Forrest plot of estimated hazard ratios on primary patency comparing Dacron ${ }^{\circledR}$ and PTFE for each study and by random-effect meta-analysis (PTFE: polytetrafluoroethylene, SE: standard error, Cl: confidence interval).

analysis might change, if more study data could be included, especially data of the trial by Jensen et al. [20] as this was the largest trial to date comparing PTFE vs. Dacron $^{\circledast}$. However, it should be noted that patient recruitment and surgeries of that trial were performed up to 14 years before publication of the results (2 year follow up). For the meta-analysis no raw data with actual times of event or censoring could be used, as only aggregated life table data was available. This will overestimate event and censoring times in both groups, but should not have influenced the comparison of the two graft materials.

In the context of clinical routine care, physicians regularly face decisions as to which alternative treatment strategies to recommend and use. Their decisions should be guided by best possible evidence of previous studies. Hence, systematic reviews of good quality RCT have evolved as important tool of decision support. It is important to note, however, that the RCT included in our systematic review were limited by a number of methodological limitations, such as rather small sample sizes, different meth- ods for determining patency rates, a lack of consideration paid to additional factors that might affect outcomes such as baseline differences between groups, and inadequate interpretations of non-significant results. Reporting of existing baseline differences between the groups in each trial was heterogeneous. Only 4 studies provided information about adjustment for baseline differences as potentially confounding factors $[13,14,18,19]$. Group differences at baseline may thus have biased the results. In 4 trials adequate sample size calculations were reported $[15,18-20]$. The other trials may have been too small to detect any differences in the graft materials. In addition, no trial used equivalence testing to show that both graft types were similarly effective. All studies described how primary patency was assessed and defined, and most studies used recommended objective standard methods [11]. However, differences in patency rates between studies might have been affected by unequal assessments, while differences in patency between PTFE or Dacron ${ }^{\circledR}$ grafts within studies should not be affected (assuming same

\begin{tabular}{|c|c|c|c|c|c|}
\hline Study or Subgroup & log[Hazard Ratio] & SE & Weight & $\begin{array}{c}\text { Hazard Ratio } \\
\text { N, Random, } 95 \% \mathrm{Cl}\end{array}$ & $\begin{array}{c}\text { Hazard Ratio } \\
\text { N, Random, } 95 \% \mathrm{Cl}\end{array}$ \\
\hline Robinson, 1999 & 0.30328 & 0.32153 & $41.2 \%$ & $1.35[0.72,2.54]$ & \\
\hline Robinson, 2003 & -0.17305 & 0.25224 & $58.8 \%$ & $0.84[0.51,1.38]$ & \\
\hline Total $(95 \% \mathrm{Cl})$ & & & $100.0 \%$ & $1.02[0.65,1.62]$ & \\
\hline \multicolumn{6}{|c|}{ 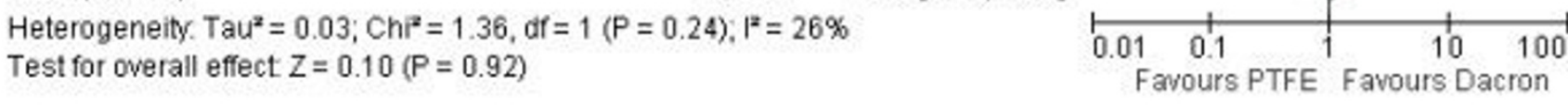 } \\
\hline
\end{tabular}

Figure 3

Forrest plot of estimated hazard ratios on secondary patency comparing Dacron ${ }^{\circledR}$ and PTFE for each study and by random-effect meta-analysis (PTFE: polytetrafluoroethylene, SE: standard error, CI: confidence interval). 


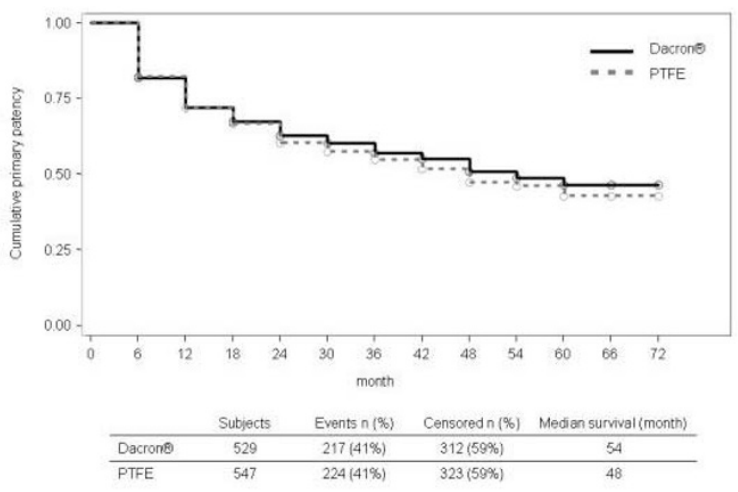

Figure 4

Survival curves for Dacron ${ }^{\circledR}$ and PTFE on primary patency of pooled data $[12-14,17, \mid 8]$ (PTFE: polytetrafluoroethylene).

assessment standards for patients receiving PTFE or Dacron $^{\circledast}$ grafts within the trial).

Sources of funding were not specified in 4 trials, with the remaining trials being funded by or having received grants from bypass graft manufacturers, which might introduce bias to the results $[23,24]$. No explicitly independent/ non-manufacturer sponsored trials were found.

In this review only trials published in English or German were considered for inclusion. Even though we feel to cover the most important and qualitatively higher trials with this strategy, this might result in relevant articles and evidence published in other languages being ignored. Results might change if a body of evidence from manuscripts in other languages would be available. Since 4 of the 9 includes trials were conducted in non-English speaking countries (Austria, Germany, and Scandinavian coun-



Figure 5

Survival curves for Dacron ${ }^{\circledR}$ and PTFE on secondary patency of pooled data [13,I7](PTFE: polytetrafluoroethylene). tries), but were published in English, we assume this might not be the case.

\section{Conclusion}

Even in the light of methodological limitations of the included trials, the present meta-analysis and systematic review might offer a basis for clinical decision making in individual patients requiring peripheral vascular surgery. Between the two prosthetic materials PTFE and Dacron ${ }^{\circledR}$ no clear advantage of one over the other could be seen. Further independently funded studies should address the issue of heparin-bonded grafts as well as the identification of subgroups of patients, in which there might be a benefit of one material. Studies should be sufficiently powered to be able to detect differences or equivalence of PTFE and Dacron $^{\circledR}$ grafts.

\section{Competing interests}

The authors declare that they have no competing interests.

\section{Authors' contributions}

SR and DE were responsible for the methodological design of the review, the literature selection and the quality assessment. SR carried out the data extraction, the summary of the findings, the meta-analysis and has participated in the writing of the manuscript. JMN has participated in the writing of the manuscript. TK and WG have revised the manuscript critically. HS advised the review as a clinical expert and has revised the manuscript critically. SW had the overall scientific responsibility and has participated in the writing of the manuscript. All authors read and approved the final manuscript.

\section{Acknowledgements}

Financial support: DIMDI (German Institute of Medical Documentation and Information)

\section{References}

I. Diehm C, Kareem S, Lawall H: Epidemiology of peripheral arterial disease. Vasa 2004, 33: I83-189.

2. Diehm C, Schuster A, Allenberg JR, Darius H, Haberl R, Lange S, et al.: High prevalence of peripheral arterial disease and comorbidity in $\mathbf{6 8 8 0}$ primary care patients: cross-sectional study. Atherosclerosis 2004, 172:95-105.

3. Ouriel K: Peripheral arterial disease. Lancet 200I, 358:1257-1264.

4. Hirsch AT, Haskal Z], Hertzer NR, Bakal CW, Creager MA, Halperin JL, et al.: ACC/AHA 2005 Practice Guidelines for the management of patients with peripheral arterial disease (lower extremity, renal, mesenteric, and abdominal aortic): a collaborative report from the American Association for Vascular Surgery/Society for Vascular Surgery, Society for Cardiovascular Angiography and Interventions, Society for Vascular Medicine and Biology, Society of Interventional Radiology, and the ACC/AHA Task Force on Practice Guidelines (Writing Committee to Develop Guidelines for the Management of Patients With Peripheral Arterial Disease): endorsed by the American Association of Cardiovascular and Pulmonary Rehabilitation; National Heart, Lung, and Blood Institute; Society for Vascular Nursing; TransAtlantic Inter-Society Consensus; and Vascular Disease Foundation. Circulation 2006, II3:e463-e654. 
5. Johnson WC, Lee KK: A comparative evaluation of polytetrafluoroethylene, umbilical vein, and saphenous vein bypass grafts for femoral-popliteal above-knee revascularization: a prospective randomized Department of Veterans Affairs cooperative study. J Vasc Surg 2000, 32:268-277.

6. Kreienberg PB, Darling RC III, Champagne BJ, Paty PS, Roddy SP, Lloyd WE, et al.: Early results of a prospective randomized trial of spliced vein versus polytetrafluoroethylene graft with a distal vein cuff for limb-threatening ischemia. J Vasc Surg 2002, 35:299-306.

7. Klinkert P, Schepers A, Burger DH, van B, Breslau PJ: Vein versus polytetrafluoroethylene in above-knee femoropopliteal bypass grafting: five-year results of a randomized controlled trial. J Vasc Surg 2003, 37: 149-155.

8. Klinkert P, Post PN, Breslau PJ, van Bockel JH: Saphenous vein versus PTFE for above-knee femoropopliteal bypass. A review of the literature. Eur J Vasc Endovasc Surg 2004, 27:357-362.

9. Sayers RD, Raptis S, Berce M, Miller JH: Long-term results of femorotibial bypass with vein or polytetrafluoroethylene. $\mathrm{Br} J$ Surg 1998, 85:934-938.

10. German Scientific Working Group Technology Assessment for Health Care (Hrsg.): Methodenmanual zur Erstellung von HTA-Berichten, 2002.

II. Rutherford RB, Baker JD, Ernst C, Johnston KW, Porter JM, Ahn S, et al.: Recommended standards for reports dealing with lower extremity ischemia: revised version. I Vasc Surg 1997, 26:517-538.

12. Johnson WC, Lee KK: Comparative evaluation of externally supported Dacron and polytetrafluoroethylene prosthetic bypasses for femorofemoral and axillofemoral arterial reconstructions. Veterans Affairs Cooperative Study \#I 4I. I Vasc Surg 1999, 30:1077-1083.

13. Robinson BI, Fletcher JP, Tomlinson P, Allen RD, Hazelton SJ, Richardson $\mathrm{A}$, et al.: A prospective randomized multicentre comparison of expanded polytetrafluoroethylene and gelatinsealed knitted Dacron grafts for femoropopliteal bypass. Cardiovascular surgery 1999, 7:214-218.

14. Green RM, Abbott WM, Matsumoto T, Wheeler JR, Miller N, Veith FJ, et al:: Prosthetic above-knee femoropopliteal bypass grafting: five-year results of a randomized trial. J Vasc Surg 2000, $31: 417-425$.

15. Post S, Kraus T, Muller-Reinartz U, Weiss C, Kortmann H, Quentmeier A, et al: Dacron vs. polytetrafluoroethylene grafts for femoropopliteal bypass: a prospective randomised multicentre trial. Eur J Vasc Endovasc Surg 200I, 22:226-23I.

16. Prager MR, Hoblaj T, Nanobashvili J, Sporn E, Polterauer P, Wagner $O$, et al:: Collagen- versus gelatine-coated Dacron versus stretch PTFE bifurcation grafts for aortoiliac occlusive disease: long-term results of a prospective, randomized multicenter trial. Surgery 2003, I34:80-85.

17. Robinson BI, Fletcher JP: Fluoropolymer coated Dacron or polytetrafluoroethylene for femoropopliteal bypass grafting: a multicentre trial. ANZ journal of surgery 2003, 73:95-99.

18. Devine C, McCollum C: Heparin-bonded Dacron or polytetrafluorethylene for femoropopliteal bypass: Five-year results of a prospective randomized multicenter clinical trial. J Vasc Surg 2004, 40:924-931.

19. Eiberg JP, Roder O, Stahl-Madsen M, Eldrup N, Qvarfordt P, Laursen $A$, et al:: Fluoropolymer-coated dacron versus PTFE grafts for femorofemoral crossover bypass: randomised trial. Eur J Vasc Endovasc Surg 2006, 32:431-438.

20. Jensen LP, Lepantalo M, Fossdal JE, Roder OC, Jensen BS, Madsen MS, et al.: Dacron or PTFE for above-knee femoropopliteal bypass. a multicenter randomised study. Eur J Vasc Endovasc Surg 2007, 34:44-49.

21. Mamode N, Scott RN: Graft type for femoro-popliteal bypass surgery. Cochrane Database Syst Rev 2000:CD00 I487.

22. Abbott WM, Green RM, Matsumoto T, Wheeler JR, Miller N, Veith FJ, et al: Prosthetic above-knee femoropopliteal bypass grafting: results of a multicenter randomized prospective trial. Above-Knee Femoropopliteal Study Group. J Vasc Surg 1997, 25:19-28.

23. Kjaergard LL, Is-Nielsen B: Association between competing interests and authors' conclusions: epidemiological study of randomised clinical trials published in the BMJ. BMJ 2002, 325:249.
24. DeAngelis CD: The influence of money on medical science. JAMA 2006, 296:996-998.

\section{Pre-publication history}

The pre-publication history for this paper can be accessed here:

http://www.biomedcentral.com/1471-2482/8/22/prepub
Publish with Bio Med Central and every scientist can read your work free of charge

"BioMed Central will be the most significant development for disseminating the results of biomedical research in our lifetime. "

Sir Paul Nurse, Cancer Research UK

Your research papers will be:

- available free of charge to the entire biomedical community

- peer reviewed and published immediately upon acceptance

- cited in PubMed and archived on PubMed Central

- yours - you keep the copyright

Submit your manuscript here:

http://www.biomedcentral.com/info/publishing_adv.asp
BioMedcentral 